\title{
CONVERGENCE OF THE IMPLICIT-EXPLICIT EULER SCHEME APPLIED TO PERTURBED DISSIPATIVE EVOLUTION EQUATIONS
}

\author{
ESKIL HANSEN AND TONY STILLFJORD
}

\begin{abstract}
We present a convergence analysis for the implicit-explicit (IMEX) Euler discretization of nonlinear evolution equations. The governing vector field of such an equation is assumed to be the sum of an unbounded dissipative operator and a Lipschitz continuous perturbation. By employing the theory of dissipative operators on Banach spaces, we prove that the IMEX Euler and the implicit Euler schemes have the same convergence order, i.e., between one half and one depending on the initial values and the vector fields. Concrete applications include the discretization of diffusion-reaction systems, with fully nonlinear and degenerate diffusion terms. The convergence and efficiency of the IMEX Euler scheme are also illustrated by a set of numerical experiments.
\end{abstract}

\section{INTRODUCTION}

The implicit-explicit (IMEX) Euler scheme is a commonly used time integrator for nonlinear evolution equations of the form

$$
\dot{u}=(f+p) u, \quad u(0)=\eta,
$$

where $f$ is an unbounded dissipative operator and the perturbation $p$ is Lipschitz continuous, with a moderately sized Lipschitz constant. The scheme can be formulated in terms of the operator

$$
S_{h}=(I-h f)^{-1}(I+h p),
$$

and the solution $u(n h)$ of the evolution equation at time $t=n h$ is then approximated by the $n$-term composition $S_{h}^{n} \eta$. The scheme constitutes an especially competitive choice when discretizing systems of equations with the structure

$$
\dot{u}_{i}=f_{i} u_{i}+p_{i}\left(u_{1}, \ldots, u_{s}\right), \quad \text { for } \quad i=1, \ldots, s .
$$

The gain of using the IMEX Euler scheme in this setting is that the coupled perturbations are handled explicitly and the implicit term decouples as

$$
\left((I-h f)^{-1} u\right)_{i}=\left(I-h f_{i}\right)^{-1} u_{i},
$$

which implies that the implicit step can be parallelized. Such systems, e.g., arise in diffusion-reaction processes and typical examples of nonlinear diffusion terms $f_{i} u_{i}$,

Received by the editor June 17, 2011 and, in revised form, January 9, 2012.

2010 Mathematics Subject Classification. Primary 65J08, 65M15, 47H06.

Key words and phrases. Implicit-explicit Euler scheme, convergence orders, nonlinear evolution equations, dissipative operators.

The work of the first author was supported by the Swedish Research Council under grant 621-2007-6227. 
which cannot be analyzed by means of the classical linear theory, are the porous medium vector field $\Delta\left(\left|u_{i}\right|^{m-1} u_{i}\right)$ and the $r$-Laplacian $\nabla \cdot\left(\left|\nabla u_{i}\right|^{r-2} \nabla u_{i}\right)$.

While investigating the properties of the solution operators for nonlinear dissipative evolution equations on Banach spaces [7], Crandall and Liggett proved that the implicit Euler scheme converges as $\mathcal{O}\left(h^{q}\right)$, with $q=1 / 2$, when the initial value $\eta$ is in the domain of $f+p$. The aim of this paper is to conduct a similar convergence analysis for the IMEX Euler scheme in the general framework of dissipative operators. The idea is to prove that the IMEX Euler approximation is within a $\mathcal{O}(h)$-surrounding of the implicit Euler scheme, i.e., both schemes share the same convergence order $1 / 2 \leq q \leq 1$. Note that a convergence of order $q<1$ is to be expected if $f+p$ has no further regularizing properties [19].

An introductional reading on the IMEX Euler scheme and higher-order generalizations can be found in the monograph [13, Section IV.4]. Earlier results in the literature include convergence and stability studies for implicit-explicit time stepping schemes applied to various semilinear evolution equations [2, 3, 8, 17]. A fully nonlinear result based on viscosity solution techniques is given in [14, where the convergence order $q=1 / 2$ is obtained for the scheme $S_{h}=\mathrm{e}^{h f}(I+h p)$ when applied to a class of nonlinear degenerate parabolic equations, including viscous Hamilton-Jacobi equations with source terms. Convergence, but without explicit order results, have also been established for a wide range of splitting schemes, e.g., Lie and Peaceman-Rachford, in the context of dissipative operators [5, 11, 15]. Further convergence results for splitting approximations of other types of problem classes are surveyed in [12, 21].

\section{Preliminaries}

Let $\mathcal{X}$ be a real Banach space, with norm $\|\cdot\|$, and introduce the (left) semi-inner product $[\cdot, \cdot]: \mathcal{X} \times \mathcal{X} \rightarrow \mathbb{R}$ defined by

$$
[u, v]=\|v\| \lim _{\varepsilon \rightarrow 0^{-}} \frac{\|v+\varepsilon u\|-\|v\|}{\varepsilon} .
$$

The semi-inner product exists for any Banach space $\mathcal{X}$ [9, p. 96], and it readily satisfies the assertions below:

(i) $[u, u]=\|u\|^{2}$;

(ii) $[\alpha u, v]=\alpha[u, v]$ for all $\alpha \geq 0$;

(iii) $[u+v, w] \leq\|u\|\|w\|+[v, w]$.

Furthermore, to any operator $g: \mathcal{D}(g) \subseteq \mathcal{X} \rightarrow \mathcal{X}$ we define the Lipschitz constant $L[g] \in[0, \infty]$ as the smallest $L$ satisfying

$$
\|g u-g v\| \leq L\|u-v\| \quad \text { for all } \quad u, v \in \mathcal{D}(g) .
$$

We will interpret (1.1) as an abstract evolution equation on $\mathcal{X}$ with a vector field $f+p$ of the following type:

Assumption 2.1. The operator $f: \mathcal{D}(f) \subseteq \mathcal{X} \rightarrow \mathcal{X}$ is m-dissipative, i.e.,

$$
[f u-f v, u-v] \leq 0 \text { for all } u, v \in \mathcal{D}(f)
$$

and the range condition $\mathcal{R}(I-h f)=\mathcal{X}$ holds for every $h>0$.

Assumption 2.2. The perturbation operator $p: \mathcal{D}(p) \subseteq \mathcal{X} \rightarrow \mathcal{X}$ is Lipschitz continuous, i.e., $L[p]<\infty$, and $\mathcal{D}(f) \subseteq \mathcal{D}(p)$. 
The key observation when using the $m$-dissipative operator framework is that the corresponding resolvent $(I-h f)^{-1}$ becomes well defined and nonexpansive, i.e.,

$$
L\left[(I-h f)^{-1}\right] \leq 1 .
$$

Note that the resolvent is nonexpansive if and only if $[f u-f v, u-v] \leq 0$, and both conditions are used in the literature when defining dissipativity. Proofs and further equivalent definitions can be found in the survey [6, Section 1]. Moreover, if both Assumptions 2.1 and 2.2 hold, then the perturbed resolvent

$$
R_{h}=(I-h(f+p))^{-1}
$$

is also well defined and Lipschitz continuous for sufficiently small values of $h$; see for example [10, Theorem 5.3]. For the sake of completeness and in order to illustrate the usage of the semi-inner product, we give short proofs of these observations.

Lemma 2.3. If Assumption 2.1 holds, then $I-h f: \mathcal{D}(f) \subseteq \mathcal{X} \rightarrow \mathcal{X}$ is bijective and $L\left[(I-h f)^{-1}\right] \leq 1$ for all $h>0$.

Proof. As $I-h f$ is already assumed to be surjective, one has for every $v_{i} \in \mathcal{X}$ an element $u_{i} \in \mathcal{D}(f)$ such that $v_{i}=(I-h f) u_{i}$ and

$$
\begin{aligned}
\left\|u_{1}-u_{2}\right\|^{2} & =\left[u_{1}-u_{2}, u_{1}-u_{2}\right]=\left[\left(v_{1}-v_{2}\right)+h\left(f u_{1}-f u_{2}\right), u_{1}-u_{2}\right] \\
& \leq\left\|v_{1}-v_{2}\right\|\left\|u_{1}-u_{2}\right\|+h\left[f u_{1}-f u_{2}, u_{1}-u_{2}\right] .
\end{aligned}
$$

The dissipativity of $f$ then yields the bound

$$
\left\|u_{1}-u_{2}\right\| \leq\left\|v_{1}-v_{2}\right\|=\left\|(I-h f) u_{1}-(I-h f) u_{2}\right\|,
$$

which implies that $I-h f$ is injective and $L\left[(I-h f)^{-1}\right] \leq 1$.

Lemma 2.4. If Assumptions 2.1 and 2.2 are valid, then $R_{h}: \mathcal{X} \rightarrow \mathcal{D}(f) \subseteq \mathcal{X}$ is well defined for all $h>0$ such that $h L[p]<1$, and $L\left[R_{h}\right] \leq(1-h L[p])^{-1}$.

Proof. Let $v \in \mathcal{X}$ be fixed and consider the operator $T_{h}: \mathcal{X} \rightarrow \mathcal{X}$ given by

$$
T_{h} w=v+h p(I-h f)^{-1} w .
$$

As $L\left[T_{h}\right] \leq h L[p] L\left[(I-h f)^{-1}\right]$, the operator $T_{h}$ is a contraction when $h L[p]<1$. By Banach fixed point theorem, there exists a unique $w \in \mathcal{X}$ such that $T_{h} w=w$, and $u=(I-h f)^{-1} w$ is then the unique solution of the equation

$$
(I-h(f+p)) u=v .
$$

As $v$ was chosen arbitrarily, the operator $I-h(f+p)$ is a bijection for every $h>0$ such that $h L[p]<1$. The Lipschitz continuity of $R_{h}$ follows as

$$
\left\|u_{1}-u_{2}\right\| \leq\left\|v_{1}-v_{2}\right\|+h L[p]\left\|u_{1}-u_{2}\right\|,
$$

where $v_{i}=(I-h(f+p)) u_{i}$. Compare with the bound (2.1).

\section{Mild solutions And the implicit Euler scheme}

Evolution equations governed by dissipative vector fields can be cast into the theory of nonlinear semigroups. The central result, originally due to [7, Theorem I], states the following: Let $g: \mathcal{D}(g) \subseteq \mathcal{X} \rightarrow \mathcal{X}$ be a nonlinear operator with the properties that $g-M I$ is dissipative, with $M \in \mathbb{R}$, and $\mathcal{R}(I-h g)=\mathcal{X}$ for all $h>0$ 
such that $h M<1$. To every such operator $g$ one can relate a nonlinear semigroup $\left\{\mathrm{e}^{t g}\right\}_{t \geq 0}$ via the limit

$$
\mathrm{e}^{t g} \eta=\lim _{m \rightarrow \infty}\left(I-\frac{t}{m} g\right)^{-m} \eta \quad \text { for all } \quad \eta \in \overline{\mathcal{D}(g)} .
$$

The nonlinear operator $\mathrm{e}^{t g}$ maps $\overline{\mathcal{D}(g)}$ into itself and $L\left[\mathrm{e}^{t g}\right] \leq \mathrm{e}^{t M}$ for every $t \geq 0$. The unique strong solution of the evolution equation governed by the operator $g$,

$$
\dot{v}=g v, \quad v(0)=\eta,
$$

can then be characterized as $v(t)=\mathrm{e}^{t g} \eta$, whenever the Banach space $\mathcal{X}$ is reflexive and $\eta \in \mathcal{D}(g)$. See for example [6. Corollary 1]. Even if the reflexivity of $\mathcal{X}$ is dropped, i.e., (3.1) might not have a strong solution, the continuous function $t \mapsto \mathrm{e}^{t g} \eta$ is still well defined and one then refers to it as a mild solution of (3.1). Surveys of the nonlinear semigroup theory can be found in [4, 6, 10,

The mild solution $v(t)=\mathrm{e}^{t g} \eta$ is in fact the limit of the implicit Euler discretization of the evolution equation (3.1). The proof of Theorem I in [7] also yields an error bound of the form

$$
\left\|(I-h g)^{-n} \eta-v(n h)\right\| \leq 2(h T)^{1 / 2} \mathrm{e}^{4 M T}\|g \eta\|, \quad 0 \leq n h \leq T,
$$

when $\eta \in \mathcal{D}(g)$ and $h M \leq 1 / 2$. Hence, the implicit Euler scheme has at least a convergence order $q=1 / 2$. The classical convergence order $q=1$ can be recovered, e.g., if one in addition assumes that $\mathcal{X}$ is a Hilbert space and the operator $g$ is the Gâteaux differential of a convex, lower semicontinuous and proper functional from $\mathcal{X}$ into $(-\infty, \infty]$, as proven in [19, Theorem 5].

\section{Convergence analysis}

Under Assumptions 2.1 and 2.2, our perturbed vector field $g=f+p$ satisfies that $g-L[p] I$ is dissipative and the range condition $\mathcal{R}(I-h g)=\mathcal{X}$ is valid for every $h>0$ such that $h L[p]<1$. This is all a direct consequence of Lemma 2.4. Hence, there exists a unique mild solution

$$
u(t)=\mathrm{e}^{t(f+p)} \eta=\lim _{m \rightarrow \infty} R_{t / m}^{m} \eta
$$

of the evolution equation (1.1), and our aim is to approximate it by employing the IMEX Euler method given in (1.2). The main difficulty of conducting an error analysis in the current context is that the mild solution lacks temporal regularity. The only feasible strategy, which does not rely on artificial regularity assumptions, is to first estimate the distance between the IMEX Euler approximation $S_{h}$ and the implicit Euler scheme $R_{h}$, and thereafter to employ the error bounds for the implicit Euler approximation. To do so, we first collect some properties of the operator $R_{h}$.

Lemma 4.1. If Assumptions 2.1 and 2.2 hold, $\eta \in \mathcal{D}(f)$ and $h L[p]<1$, then the resolvent $R_{h}$ satisfies

(i) $\left\|R_{h}^{n} \eta-R_{h}^{n-1} \eta\right\| \leq h(1-h L[p])^{-n}\|(f+p) \eta\| \quad$ and

(ii) $\left\|R_{h}^{n} \eta-\eta\right\| \leq n h(1-h L[p])^{-n}\|(f+p) \eta\|$

for every positive integer $n$.

Proof. We first observe that

$$
\left\|R_{h} \eta-\eta\right\|=\left\|R_{h} \eta-R_{h}(I-h(f+p)) \eta\right\| \leq h(1-h L[p])^{-1}\|(f+p) \eta\|,
$$


and assertion (i) now follows as

$$
\left\|R_{h}^{n} \eta-R_{h}^{n-1} \eta\right\| \leq(1-h L[p])^{-n+1}\left\|R_{h} \eta-\eta\right\| \leq h(1-h L[p])^{-n}\|(f+p) \eta\| .
$$

A direct consequence of (i) is the bound

$$
\begin{aligned}
\left\|R_{h}^{n} \eta-\eta\right\| & \leq \sum_{k=1}^{n}\left\|R_{h}^{k} \eta-R_{h}^{k-1} \eta\right\| \leq h \sum_{k=1}^{n}(1-h L[p])^{-k}\|(f+p) \eta\| \\
& \leq n h(1-h L[p])^{-n}\|(f+p) \eta\|,
\end{aligned}
$$

which proves assertion (ii).

With this in place, we can give an estimate of the distance between the two schemes.

Lemma 4.2. If Assumptions 2.1 and 2.2 hold, $\eta \in \mathcal{D}(f)$ and $h L[p] \leq 1 / 2$, then

$$
\left\|S_{h}^{n} \eta-R_{h}^{n} \eta\right\| \leq h T L[p] \mathrm{e}^{2 T L[p]}\|(f+p) \eta\|, \quad 0 \leq n h \leq T .
$$

Proof. In order to shorten the notation we introduce

$$
\begin{array}{lll}
u_{n}=S_{h} u_{n-1}=u_{n-1}+h f u_{n}+h p u_{n-1}, & u_{0}=\eta, & \text { and } \\
v_{n}=R_{h} v_{n-1}=v_{n-1}+h f v_{n}+h p v_{n}, & v_{0}=\eta . &
\end{array}
$$

We can then bound the difference $S_{h}^{n} \eta-R_{h}^{n} \eta=u_{n}-v_{n}$ as

$$
\begin{aligned}
\left\|u_{n}-v_{n}\right\|^{2}= & {\left[u_{n}-v_{n}, u_{n}-v_{n}\right] } \\
= & {\left[\left(u_{n-1}-v_{n-1}\right)+h\left(p u_{n-1}-p v_{n}\right)+h\left(f u_{n}-f v_{n}\right), u_{n}-v_{n}\right] } \\
\leq & \left(\left\|u_{n-1}-v_{n-1}\right\|+h L[p]\left\|u_{n-1}-v_{n}\right\|\right)\left\|u_{n}-v_{n}\right\| \\
& \quad+h\left[f u_{n}-f v_{n}, u_{n}-v_{n}\right] \\
\leq & \left((1+h L[p])\left\|u_{n-1}-v_{n-1}\right\|+h L[p]\left\|v_{n}-v_{n-1}\right\|\right)\left\|u_{n}-v_{n}\right\| .
\end{aligned}
$$

By the above bound and Lemma 4.1(i) we obtain that

$$
\begin{aligned}
\left\|u_{n}-v_{n}\right\| & \leq(1+h L[p])\left\|u_{n-1}-v_{n-1}\right\|+h^{2} L[p](1-h L[p])^{-n}\|(f+p) \eta\| \\
& \leq \mathrm{e}^{h L[p]}\left\|u_{n-1}-v_{n-1}\right\|+h^{2} L[p] \mathrm{e}^{2 n h L[p]}\|(f+p) \eta\|,
\end{aligned}
$$

where the last inequality follows as $(1-h L[p])^{-1} \leq \mathrm{e}^{2 h L[p]}$ for all $h L[p] \leq 1 / 2$. Note that $u_{0}=v_{0}$ and $0 \leq n h \leq T$. Hence, an $n$-fold repetition of the above argument then yields

$$
\left\|u_{n}-v_{n}\right\| \leq h^{2} L[p] \sum_{k=1}^{n} \mathrm{e}^{(n+k) h L[p]}\|(f+p) \eta\| \leq h T L[p] \mathrm{e}^{2 T L[p]}\|(f+p) \eta\|,
$$

and the sought after bound is obtained.

Combining Lemma 4.2 with the error bound (3.2) for the implicit Euler scheme yields the following new convergence result for the IMEX Euler approximation of the mild solution, without assuming any extra temporal regularity:

Theorem 4.3. Consider the implicit-explicit Euler approximation (1.2) of the mild solution (4.1). If Assumptions 2.1 and 2.2 hold, $\eta \in \mathcal{D}(f)$ and $h L[p] \leq 1 / 2$, then the implicit-explicit Euler scheme has the same convergence order $q \in[1 / 2,1]$ as the implicit Euler scheme, and

$$
\left\|S_{h}^{n} \eta-u(n h)\right\| \leq C(T, \eta)\left(h^{q}+h\right), \quad 0 \leq n h \leq T .
$$


With the partition $\{0, h, \ldots, N h=T\}$ of the bounded time interval $[0, T]$, the above convergence result can also be stated in terms of the piecewise constant function $u_{h}:[0, T] \rightarrow \mathcal{D}(f) \subseteq \mathcal{X}$, where

$$
u_{h}(t)=S_{h}^{n} \eta \quad \text { for } \quad t \in((n-1) h, n h] \text { and } u_{h}(0)=\eta \text {. }
$$

Corollary 4.4. Under the assumptions of Theorem 4.3, the family of piecewise constant functions (4.2) approximates the mild solution (4.1) with the same convergence order $q \in[1 / 2,1]$ as the implicit Euler scheme, and

$$
\left\|u_{h}-u\right\|_{L^{\infty}(0, T ; \mathcal{X})} \leq C(T, \eta)\left(h^{q}+h\right) .
$$

Proof. Consider the partition $\{0, h, \ldots, N h=T\}$. For every $t \in[0, T]$ there is then a nonnegative integer $n$ and a $\tau \in[0, h)$ such that $t=n h+\tau$. Hence, by Theorem 4.3. we have

$$
\begin{aligned}
\left\|u_{h}(t)-u(t)\right\| & =\left\|S_{h}^{n} \eta-u(t)\right\| \leq\left\|S_{h}^{n} \eta-u(n h)\right\|+\|u(n h+\tau)-u(n h)\| \\
& \leq C(T, \eta)\left(h^{q}+h\right)+\|u(n h+\tau)-u(n h)\| .
\end{aligned}
$$

Next, the semigroup property and the Lipschitz continuity of $\mathrm{e}^{t(f+p)}$ together with Lemma 4.1(ii) imply that

$$
\begin{aligned}
\|u(n h+\tau)-u(n h)\| & =\left\|\mathrm{e}^{n h(f+p)} \mathrm{e}^{\tau(f+p)} \eta-\mathrm{e}^{n h(f+p)} \eta\right\| \\
& \leq \mathrm{e}^{n h L[p]} \lim _{m \rightarrow \infty}\left\|R_{\tau / m}^{m} \eta-\eta\right\| \\
& \leq \mathrm{e}^{n h L[p]} \lim _{m \rightarrow \infty} \tau\left(1-\frac{\tau}{m} L[p]\right)^{-m}\|(f+p) \eta\| \\
& =\tau \mathrm{e}^{t L[p]}\|(f+p) \eta\| \leq h \mathrm{e}^{T L[p]}\|(f+p) \eta\| .
\end{aligned}
$$

The $L^{\infty}(0, T ; \mathcal{X})$-bound then follows as $t$ was chosen arbitrarily in $[0, T]$.

\section{Applications}

5.1. Nonlinear equation systems. As already stated in the introduction, the IMEX Euler scheme constitutes a competitive method choice when applied to nonlinear systems with the form given in (1.3). These systems can be interpreted as abstract evolution equations (1.1) with an operator $f: \mathcal{D}(f) \subseteq \mathcal{X} \rightarrow \mathcal{X}$ having the structure

$$
(f u)_{i}=f_{i} u_{i}, \quad \text { for } i=1, \ldots, s,
$$

where $\mathcal{X}=\left(\mathcal{X}_{1} \times \ldots \times \mathcal{X}_{s} ;\|\cdot\|=\sum_{i=1}^{s}\|\cdot\|_{\mathcal{X}_{i}}\right)$ and $f_{i}: \mathcal{D}\left(f_{i}\right) \subseteq \mathcal{X}_{i} \rightarrow \mathcal{X}_{i}$. If the operators $f_{i}$ are $m$-dissipative, i.e., fulfilling Assumption 2.1, then the same holds true for the full $f$.

Concrete examples of such systems occur in the context of nonlinear diffusionreaction processes and two standard diffusion terms $f_{i} u_{i}$ are the porous medium vector field

and the $r$-Laplacian

$$
f_{i} u_{i}=\Delta\left(\left|u_{i}\right|^{m-1} u_{i}\right), \quad m \geq 1,
$$

$$
f_{i} u_{i}=\nabla \cdot\left(\left|\nabla u_{i}\right|^{r-2} \nabla u_{i}\right), \quad r \geq 2 .
$$

If we consider a bounded domain $\Omega$, with a sufficiently regular boundary, and homogeneous Dirichlet boundary conditions, then the porous medium vector field becomes $m$-dissipative in both $H^{-1}(\Omega)$ and $L^{1}(\Omega)$, and the $r$-Laplacian is $m$ dissipative in $L^{2}(\Omega)$. Proofs and several other examples can be found in 4, Chapters 2 and 3], [18, Chapters 3 and 4] and [22, Chapter 10]. 
5.2. Locally Lipschitz continuous perturbations. Assumption 2.2, concerning the Lipschitz continuity of the perturbation $p$, can actually be weakened when the Banach space $\mathcal{X}$ has more structure. For example, assume that $\mathcal{X}$ is reflexive and the perturbation $p: \mathcal{X} \rightarrow \mathcal{X}$ is locally Lipschitz continuous around the initial value $\eta \in \mathcal{D}(f)$, i.e., for every $r>0$ there exists a constant $L_{r}[p]<\infty$ such that

$$
\|p u-p v\| \leq L_{r}[p]\|u-v\| \quad \text { for all } u, v \in B_{r}(\eta),
$$

where $B_{r}(\eta)=\{u \in \mathcal{X}:\|u-\eta\| \leq r\}$. Next, consider the truncation [4, p. 150]

$$
p_{r} u= \begin{cases}p u & \text { if } \quad\|u-\eta\| \leq r \\ p\left(\frac{r}{\|u-\eta\|}(u-\eta)+\eta\right) & \text { if } \quad\|u-\eta\|>r .\end{cases}
$$

The new operator $p_{r}: \mathcal{X} \rightarrow \mathcal{X}$ is Lipschitz continuous, with

$$
L\left[p_{r}\right] \leq 2 L_{r}[p] .
$$

As $\mathcal{X}$ was assumed to be reflexive, the function $u_{r}(t)=\mathrm{e}^{t\left(f+p_{r}\right)} \eta$ is the unique strong solution of the evolution equation

$$
\dot{u}_{r}=(f+p) u_{r}, \quad u_{r}(0)=\eta,
$$

for all times $t \in[0, T(\eta)]$ such that $u_{r}(t) \in B_{r}(\eta)$. In order to characterize $T(\eta)$ we observe that

$$
\left\|\mathrm{e}^{t\left(f+p_{r}\right)} \eta-\eta\right\| \leq \lim _{m \rightarrow \infty}\left\|R_{t / m}^{m} \eta-\eta\right\| \leq t \mathrm{e}^{2 t L_{r}[p]}\|(f+p) \eta\|,
$$

where the last inequality follows by Lemma 4.1(ii). Hence, $u_{r}(t)$ remains in $B_{r}(\eta)$ for all times $t$ such that $t \mathrm{e}^{2 t L_{r}[p]}\|(f+p) \eta\| \leq r$, and $T(\eta)$ can then be bounded from below in terms of the Lambert $W$ function:

$$
T(\eta) \geq \sup _{r>0} \frac{1}{2 L_{r}(p)} W\left(\frac{2 L_{r}(p) r}{\|(f+p) \eta\|}\right) .
$$

If $\mathcal{X}$ is also a Hilbert space, then one may consider locally Lipschitz continuous perturbations which are only defined on $\mathcal{D}(f)$. In this case the Lipschitz continuous extension $p_{r}: \mathcal{X} \rightarrow \mathcal{X}$, with $L\left[p_{r}\right]=L_{r}[p]$, can be obtained from Kirszbraun's lemma [20, Theorem 1.31] and the lower bound of $T(\eta)$ is improved, as the factors of 2 in (5.1) are avoided.

\section{NumericAl EXPERIMENTS}

6.1. Efficiency and convergence for a nonlinear system. To illustrate the efficiency of the IMEX Euler scheme, in comparison to the implicit Euler scheme, we consider a diffusion-reaction system with the vector field $f+p$ defined as

$$
\begin{array}{lll}
(f u)_{1}=d_{1} \Delta u_{1}, & (p u)_{1}=\alpha_{1} u_{1}\left(1-u_{1} / \gamma\right)-\beta_{1} u_{1} u_{2}, \\
(f u)_{2}=d_{2} \Delta u_{2}, & (p u)_{2}=\alpha_{2} u_{1} u_{2}-\beta_{2} u_{2}, \\
(f u)_{3}=\Delta\left(\left|u_{3}\right|^{1 / 2} u_{3}\right), & (p u)_{3}=\alpha_{3}\left(u_{1}+u_{2}\right) u_{3}-\beta_{3} u_{3},
\end{array}
$$

where $d_{1}=0.01, d_{2}=0.02, \alpha=(1,20,100), \beta=(5,1,50)$ and $\gamma=0.25$. This represents a prey-predator-parasite model, where $u_{1}$ and $u_{2}$ are the densities of prey and predators respectively. The third component, $u_{3}$, represents the density of parasites that feed on both the prey and on the predators. The species interact according to standard Lotka-Volterra population dynamics. The prey and predators are assumed to disperse in a random walk fashion throughout the two-dimensional habitat, whereas the dispersion of the airborne parasites requires a more complex 
model. In the case of mosquito swarms, Okubo [16] suggests that the dispersion process is governed by the porous medium operator, with $m=3 / 2$.

The system is given over the unit square $\Omega=(0,1)^{2}$ together with homogeneous Dirichlet boundary conditions. If we interpret the system as an evolution equation (1.1) on the Banach space $\mathcal{X}=C(\bar{\Omega}) \times C(\bar{\Omega}) \times L^{1}(\Omega)$, then the operator $f$ becomes $m$-dissipative. Indeed, the porous medium operator is $m$-dissipative on $L^{1}(\Omega)$ as mentioned in Section 5] The Laplacian with Dirichlet boundary conditions on $C(\bar{\Omega})$ is also $m$-dissipative as proven in [1, pp. 12-14]. Note that the verification of the $m$-dissipativity requires a rather lengthy investigation in both cases, and we therefore omit the proofs. Furthermore, the local Lipschitz continuity of the perturbation $p: \mathcal{X} \rightarrow \mathcal{X}$ follows as $C(\bar{\Omega}) \subset L^{\infty}(\Omega)$ together with the estimates

$$
\begin{aligned}
\left\|u_{j} u_{k}-v_{j} v_{k}\right\|_{C(\bar{\Omega})} & \leq\left\|u_{j}\right\|_{C(\bar{\Omega})}\left\|u_{k}-v_{k}\right\|_{C(\bar{\Omega})}+\left\|v_{k}\right\|_{C(\bar{\Omega})}\left\|u_{j}-v_{j}\right\|_{C(\bar{\Omega})} \quad \text { and } \\
\left\|u_{j} u_{3}-v_{j} v_{3}\right\|_{L^{1}(\Omega)} & \leq\left\|u_{j}\right\|_{L^{\infty}(\Omega)}\left\|u_{3}-v_{3}\right\|_{L^{1}(\Omega)}+\left\|v_{3}\right\|_{L^{1}(\Omega)}\left\|u_{j}-v_{j}\right\|_{L^{\infty}(\Omega)},
\end{aligned}
$$

where $j, k=1,2$.

To solve the problem numerically, we discretize it by standard central differences over an equidistant grid with $N \times N$ grid points for each of the three components of the solution. We take $N=150$. With $\Delta x=1 /(N+1)$, the Laplacian is then represented by the matrix $T$ having the value $-4 / \Delta x^{2}$ on the main diagonal and $1 / \Delta x^{2}$ on the first and $N$ th sub- and super-diagonals. The action of the porous medium operator on the vector $u$ is represented by $T v$, where $v_{i}=\left|u_{i}\right|^{1 / 2} u_{i}$. The solutions of the algebraic equations, obtained when evaluating the nonlinear implicit parts of the time stepping schemes, are approximated by Newton's method. We finally choose the initial value $\eta$ to have the components

$$
\eta_{1}=\sum_{k=1}^{4} \mathrm{e}^{-150\left|x-x_{1, k}\right|^{2}}, \quad \eta_{2}=\mathrm{e}^{-100\left|x-x_{2}\right|^{2}}, \quad \eta_{3}=\left[1-800 / 9\left|x-x_{3}\right|^{2}\right]_{+}^{2},
$$

where $\left(x_{1,1} ; x_{1,2} ; x_{1,3} ; x_{1,4}\right)=((0.35,0.35) ;(0.35,0.65) ;(0.65,0.35) ;(0.65,0.65)), x_{2}=$ $(0.57,0.57), x_{3}=(0.55,0.61)$ and $[\cdot]_{+}=\max \{\cdot, 0\}$. Note that $\eta_{3}$ is the analytic solution to the homogeneous problem $\dot{u}=\alpha \Delta\left(|u|^{1 / 2} u\right)$ at time $t=1$, with a weighted and translated Dirac delta as initial value [22, p. 5], which ensures that $\eta$ is an element of $\mathcal{D}(f)$.

The temporal errors at time $t=n h=0.1$ are then computed for varying time steps sizes $h$ in the discrete $\mathcal{X}$-norm. We use the implicit Euler approximation, with $h=2^{-11}$, as a reference solution. The resulting errors and the execution times are given in Figure 1. The results show that both the IMEX Euler and the implicit Euler schemes have the convergence order $q=1$. Furthermore, we observe that for a given accuracy the effort to compute the IMEX Euler approximation is significantly less than for the implicit Euler scheme, i.e., the IMEX Euler method is indeed more efficient in the current context. It should be noted that this increase in performance is without any parallellization of the procedure, hence even better performance is possible.

6.2. Optimal convergence orders. In order to show that convergence of the form $\mathcal{O}\left(h^{q}\right)$, with $1 / 2 \leq q<1$, is to be expected in general for the IMEX Euler and the implicit Euler schemes, we look at the following problem: Let $\mathcal{X}=\ell^{2}$ and 

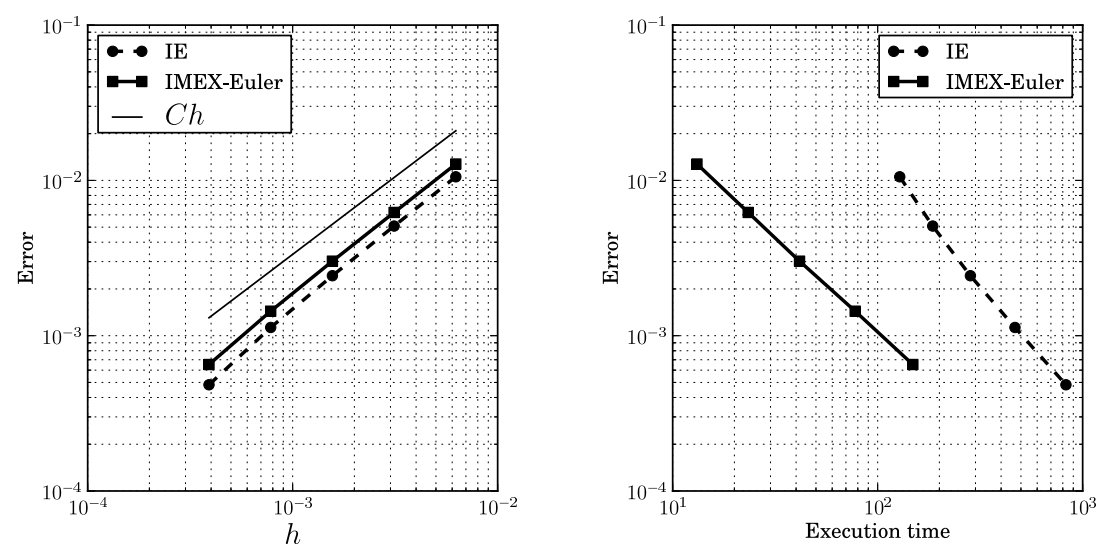

Figure 1. The errors for the problem described in Section 6.1 in the discrete $\mathcal{X}$-norm plotted against the step size $h$ (left) and against the execution time (right).

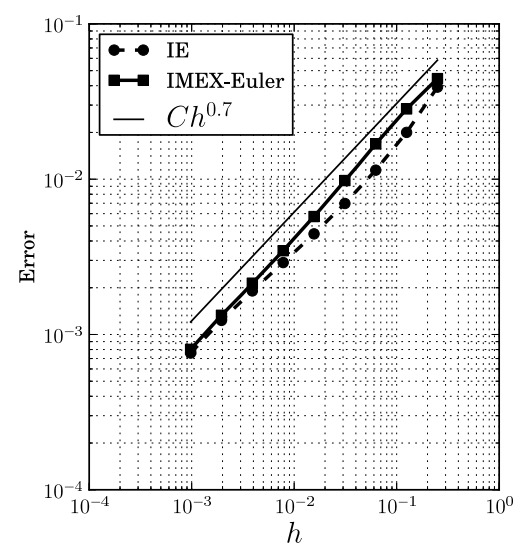

Figure 2. Truncated $\ell^{2}$-errors at time $t=1$ for varying time step sizes $h$, when applied to the problem described in Section 6.2

consider the linear evolution equation (1.1) governed by $f+p$, where

$$
\begin{aligned}
f\left(u_{1}, u_{2}, \ldots, u_{2 k-1}, u_{2 k}, \ldots\right) & =\left(u_{2},-u_{1}, \ldots, k u_{2 k},-k u_{2 k-1}, \ldots\right) \quad \text { and } \\
p\left(u_{1}, u_{2}, u_{3}, \ldots\right) & =\left(u_{1}, u_{2}+u_{1}, u_{3}+u_{2}, \ldots\right) .
\end{aligned}
$$

This particular $f$ is chosen as it is proven in [19, Example 3] that the implicit Euler scheme has a convergence order $q<1$ for the unperturbed problem given that $\eta \in \mathcal{D}(f) \backslash \mathcal{D}\left(f^{2}\right)$. The reason for this order reduction is that the analytical solution lacks temporal regularity for such a rough initial value. As the semi-inner product coincides with the inner product on a Hilbert space, two straightforward 
calculations yields that the operator $f$ is $m$-dissipative. The operator $p: \mathcal{X} \rightarrow \mathcal{X}$ is (globally) Lipschitz continuous, as it is the sum of the identity and the right shift operator.

For our numerical experiment we truncate all $\ell^{2}$-series after 1000 components and the errors are computed at time $t=n h=1$ for different time step sizes $h$. The reference solution is again given by the implicit Euler approximation, with $h=2^{-12}$. For the initial value $\eta=\left\{1 / k^{1.51}\right\}_{k=1}^{\infty} \in \mathcal{D}(f) \backslash \mathcal{D}\left(f^{2}\right)$ one obtains the errors presented in Figure 2. As seen from the experiment, both the IMEX Euler and the implicit Euler schemes converge with an order $q=0.7$, i.e., in full agreement with Theorem 4.3 ,

\section{REFERENCES}

[1] W. Arendt, Semigroups and evolution equations: functional calculus, regularity and kernel estimates, Handb. Differ. Equ., vol. I , North-Holland, Amsterdam, 2004, pp. 1-85. MR2103696 (2005j:47041)

[2] G. Akrivis, M. Crouzeix and C. Makridakis, Implicit-explicit multistep methods for quasilinear parabolic equations, Numer. Math. 82 (1999) 521-541. MR1701828 (2000e:65075)

[3] G. Akrivis and M. Crouzeix, Linearly implicit methods for nonlinear parabolic equations, Math. Comp. 73 (2004) 613-635. MR2031397 (2005a:65097)

[4] V. Barbu, Nonlinear Differential Equations of Monotone Types in Banach spaces, Springer, New York, 2010. MR2582280(2011d:34001)

[5] H. Brézis and A. Pazy, Convergence and approximation of semigroups of nonlinear operators in Banach spaces, J. Funct. Anal. 9 (1972) 63-74. MR0293452 (45:2529)

[6] M.G. Crandall, Nonlinear semigroups and evolution governed by accretive operators, Proc. Sympos. Pure Math., vol. 45, Amer. Math. Soc., Providence, RI, 1986, pp. 305-337. MR.843569 (87h:47140)

[7] M.G. Crandall and T.M. Liggett, Generation of semi-groups of nonlinear transformations on general Banach spaces, Amer. J. Math. 93 (1971) 265-298. MR0287357 (44:4563)

[8] M. Crouzeix, Une méthode multipas implicite-explicite pour l'approximation des équations d'évolution paraboliques, Numer. Math. 35 (1980) 257-276. MR592157 (82b:65084)

[9] K. Deimling, Nonlinear Functional Analysis, Springer, Berlin, 1985. MR.787404 (86j:47001)

[10] G.R. Goldstein, Nonlinear semigroups and applications, Proc. of the Curaçao Conference, August 1992, Kluwer Acad. Publ., Dordrecht, 1993, pp. 59-98. MR.1270691 (95c:47081)

[11] E. Hansen and A. Ostermann, Dimension splitting for quasilinear parabolic equations, IMA J. Numer. Anal. 30 (2010) 857-869. MR2670117(2011j:65109)

[12] H. Holden, K.H. Karlsen, K.A. Lie and N.H. Risebro, Splitting methods for partial differential equations with rough solutions. Analysis and MATLAB programs, EMS, Zürich, 2010. MR2662342(2011j:65002)

[13] W. Hundsdorfer and J. Verwer, Numerical solution of time-dependent advection-diffusionreaction equations, Springer, Berlin, 2003. MR2002152(2004g:65001)

[14] E.R. Jakobsen and K.H. Karlsen, Convergence rates for semi-discrete splitting approximations for degenerate parabolic equations with source terms, BIT 45 (2005) 37-67. MR2164225 (2006h:65135)

[15] P.L. Lions and B. Mercier, Splitting algorithms for the sum of two nonlinear operators, SIAM J. Numer. Anal. 16 (1979) 964-979. MR551319 (81g:47070)

[16] A. Okubo, Diffusion and Ecological Problems: Mathematical Models, Springer, Berlin, 1980. MR.572962(81i:92025)

[17] A. Ostermann, Stability of $W$-methods with applications to operator splitting and to geometric theory, Appl. Numer. Math. 42 (2002) 353-366. MR 1921347 (2003g:65130)

[18] T. Roubíček, Nonlinear partial differential equations with applications, Birkhäuser, Basel, 2005. MR2176645 (2007e:35002)

[19] J. Rulla, Error analysis for implicit approximations to solutions to Cauchy problems, SIAM J. Numer. Anal. 33 (1996) 68-87. MR.1377244 (97c:65151)

[20] J.T. Schwartz, Nonlinear Functional Analyis, Gordon and Breach Science Publishers, New York, 1969. MR0433481 (55:6457) 
[21] R. Temam, Sur la stabilité et la convergence de la méthode des pas fractionnaires, Ann. Mat. Pura Appl. (4) 79 (1968) 191-379. MR0241838(39:3175)

[22] J.L. Vázquez, The porous medium equation. Mathematical theory, Oxford University Press, Oxford, 2007. MR2286292(2008e:35003)

Centre for Mathematical Sciences, Lund University, P.O. Box 118, SE-22100 Lund, Sweden

E-mail address: eskil@maths.1th.se

Centre for Mathematical Sciences, Lund University, P.O. Box 118, SE-22100 Lund, SWEDEN

E-mail address: tony@maths.1th.se 